\title{
TÍCH HỢP IFRS VÀO CHƯƠNG TRÌNH ĐÀO TẠO CHUYÊN NGÀNH KẾ TOÁN, KIỂM TOÁN CỦA TRƯỜNG ĐẠI HỌC TÀI CHÍNH - MARKETING
}

\author{
INTEGRATING INTERNATIONAL FINANCIAL \\ REPORTING STANDARDS INTO THE ACCOUNTING \\ AND AUDITING CURRICULUM OF THE UNIVERSITY \\ OF FINANCE AND MARKETING
}

\section{Dương Hoàng Ngọc Khuê, Nguyễn Thị Ngọc Oanh, Ung Hiển Nhã Thi ${ }^{1}$}

Ngày nhận bài: 19/9/2019 Ngày chấp nhận đăng: 09/10/2019 Ngày đăng: 05/06/2020

\section{Tóm tắt}

Mục tiêu của nghiên cứu là đánh giá quá trình tích hợp các chuẩn mực báo cáo tài chính (BCTC) quốc tế (IFRS) vào chương trình đào tạo (CTĐT) chuyên ngành kế toán, kiểm toán của trường Đại học Tài chính - Marketing và đánh giá nhận thức của giảng viên, sinh viên của Khoa Kế toán - Kiểm toán về vấn đề này, đồng thời nghiên cứu cũng phân tích các nguồn lực cần thiết cho quá trình tích hợp IFRS vào chương trình đào tạo. Nghiên cứu đã sử dụng phương pháp phỏng vấn với bảng hỏi được xây dựng dựa trên lý thuyết nền CIF và các nghiên cứu trước để đánh giá các yếu tố ảnh hưởng đến quá trình tích hợp IFRS vào chương trình đào tạo gồm: yếu tố giai đoạn tích hợp IFRS, yếu tố phương pháp giảng dạy, yếu tố nhận thức về rào cản, yếu tố tổ chức bên ngoài. Mẫu nghiên cứu gồm 32 giảng viên và 142 sinh viên của khoa Kế toán - Kiểm toán trường Đại học Tài chính - Marketing. Kết quả nghiên cứu và hàm ý chính sách được thực hiện dựa trên các yếu tố như trong lý thuyết nền CIF.

Tù khóa: Chương trình đào tạo, kế toán - kiểm toán, IFRS, lý thuyết nền CIF.

\begin{abstract}
The objective of the study is to review the process of integrating international financial reporting standards (IFRS) into the accounting and auditing curriculum of the University of Finance and Marketing and assess the awareness of lecturers and students of the Faculty of Accounting and Auditing (FAA) on this issue, and the paper also analyzes the resources required for IFRS integration into the Accounting and Auditing training program.

The study used the interview method with the questionnaire built on the CIF framework and previous research, which evaluated factors that impact the integration of IFRS into the training program, including IFRS integrated stage, teaching methodology, awareness of barriers, and external organizations. The research sample consisted of 32 lecturers and 142 students from UFM's accounting and auditing department. Research results and policy implications are formed based on elements of the CIF background theory.
\end{abstract}

Keywords: Curriculum, Accounting and Auditing, IFRS, CIF framework.

${ }^{1}$ Trường Đại học Tài chính - Marketing 


\section{Giới thiệu}

Trong bối cảnh toàn cầu hóa hiện nay, yêu cầu về hội tụ kế toán quốc tế ngày càng quan trọng, vì vậy bộ chuẩn mực báo cáo tài chính (BCTC) quốc tế IAS/IFRS đã ngày càng chấp nhận rộng rãi trên thế giới. Tại Việt Nam, "Chiến lược kế toán - kiểm toán đến năm 2020, tầm nhìn năm 2030" được Thủ tướng Chính phủ phê duyệt theo Quyết định số 480/2013/QĐ-TTg đã xác định quan điểm là "hoàn thiện khuôn khổ pháp lý hệ thống chuẩn mực", "hòa hợp với thông lệ quốc tế". Theo định hướng của Bộ Tài chính, dự kiến đến 2020, chuẩn mực kế toán Việt Nam (VAS) phải được ban hành mới trên cơ sở cập nhật những thay đồi của IFRS. Hiệu lực của VAS/VFRS mới sẽ được bắt đầu từ $1 / 1 / 2020$. Trong đó, các đơn vị có lợi ích công chúng sẽ dần chuyển đổi từ VAS/VFRS sang IFRS và đến 2025 phải hoàn tất quá trình chuyển đổi. Do đó, nhu cầu đào tạo các kế toán viên có trình độ chuyên môn và hiểu biết về IFRS là yêu cầu tất yếu của các trường đại học và cao đẳng hiện nay, điều này đòi hỏi các cơ sở đào tạo phải xây dựng chương trình đào tạo (CTĐT) và phương pháp đào tạo kế toán phù hợp với IFRS nhằm cung cấp nguồn nhân lực có chất lượng cho xã hội. Do đó, việc nghiên cứu tích hợp IFRS vào CTĐT chuyên ngành kế toán, kiểm toán - nghiên cứu thực nghiệm tại trường Đại học Tài chính - Marketing là cần thiết.

\section{Cơ sở lý thuyết}

\subsection{Vài nét về IFRS}

Giữa những năm 1973 và năm 2000, các nguyên tắc kế toán được biểu hiện là chuẩn mực kế toán quốc tế (IAS) được phát hành. Từ năm 2001, Hội đồng chuẩn mực kế toán quốc tế (IASB) mô tả các nguyên tắc kế toán với tên gọi mới là Chuẩn mực lập BCTC quốc tế, mặc dù các chuẩn mực kế toán quốc tế vẫn tiếp tục được thừa nhận.

Mục tiêu hình thành bộ IFRS của IASB là cung cấp một khuôn khổ quốc tế về cách lập và trình bày $\mathrm{BCTC}$ cho các công ty đại chúng nhằm phục vụ cho lợi ích chung, chất lượng cao, dễ hiểu và có thể áp dụng trên toàn thế giới và yêu cầu thông tin trên $\mathrm{BCTC}$ phải rõ ràng, có thể so sánh, nhằm giúp những người tham gia vào các thị trường vốn khác nhau trên thế giới cũng như các đối tượng sử dụng thông tin khác ra quyết định kinh tế. IFRS tập trung vào các hướng dẫn, diễn giải chung nhất về cách lập BCTC hơn là thiết lập các quy tắc lập báo cáo ngành cụ thể. Tính đến thời điểm hiện nay, hệ thống chuẩn mực quốc tế gồm 25 IAS và 17 IFRS (IFRS in your pocket, 2019). Trong bài viết này, nhóm tác giả sử dụng thuật ngữ tích hợp IFRS bao hàm tích hợp IAS và IFRS.

\subsection{Lý thuyết nền cho việc tích hợp IFRS trong chuơng trình đào tạo kế toán - kiểm toán}

Nhiều nhà nghiên cứu như Carr và Mathews (2004), Rebele (2002) đều cho rằng các CTĐT kế toán cần phải đáp ứng với những thay đổi xảy ra trong môi trường, cho phép họ tận dụng các cơ hội mới và tránh những trở ngại tiềm năng. Tuy nhiên, do tính chất ngành nghề kế toán, việc thay đồi chương trình giảng dạy kế toán chịu ảnh hưởng của các yếu tố bên trong và bên ngoài như người học hay các cơ quan quản lý chuyên môn (Wolf và Hughes, 2007). Điều này cho thấy việc xây dựng hoặc thay đổi một CTĐT cần phải xem xét các yếu tố môi trường liên quan. Nhận định này cũng đã được chứng minh trong nghiên cứu của Fuller (2007), khi tác giả đề cập lý do thất bại của các nỗ lực cải thiện và thay đổi chương trình giảng dạy là do mức độ liên kết thấp của các mục tiêu cũng như sự hiểu biết giữa những người lập kế hoạch, thiết kế thay đổi và những người thực hiện thay đổi. Năm 2015, Albader (2015) đã giới thiệu 4 khuôn khổ về giáo dục và CTĐT để đảm bảo mức độ tập trung vào quá trình thay đổi và thực hiện, gồm: (1) mô hình Học viện Giáo dục Đại học Vương quốc Anh, (2) mô hình chương trình giảng dạy 3P của Biggs (1989, 2003), (3) mô hình khung kỷ luật ba khía cạnh (three-domains discipline) của Barnett và Coate (2005), và (4) Khuôn khổ thực hiện CTĐT (Curriculum Implementation Framework - CIF) của Rogan và Grayson (2003). 
Trong quá trình chuyển đổi sang IFRS, các trường đại học, cao đẳng tại Việt Nam sẽ cần điều chỉnh chương trình giảng dạy hiện tại của họ nhằm tích hợp IFRS. Mức độ và khả năng tích hợp sẽ phụ thuộc vào các yếu tố cụ thể của tổ chức như quy mô của tổ chức, nguồn lực hiện có, kinh nghiệm và số lượng nhân viên. Do đó, việc xây dựng một khuôn khổ hướng dẫn cho việc thiết kế, thực hiện thay đổi CTĐT theo hướng tích hợp IFRS là điều cần thiết. Trong nghiên cứu này, nhóm tác giả sử dụng khuôn khổ thứ 4 của Rogan và Grayson (2003).

Rogan và Grayson (2003) đã đề xuất một lý thuyết về việc thực hiện chương trình giảng dạy dựa trên ba cấu trúc chính, đó là "hồ sơ thực hiện", "nguồn lực hỗ trợ đồi mới", "hỗ trợ từ các tổ chức bên ngoài”. Các cấu trúc này có chung ba đặc điểm quan trọng: (i) chúng có thể được đo lường bằng các chỉ số, (ii) chúng có thể bao gồm nhiều yếu tố liên quan nhưng vẫn phản ánh đầy đủ nội dung của yếu tố chính (Rogan và Grayson, 2003).

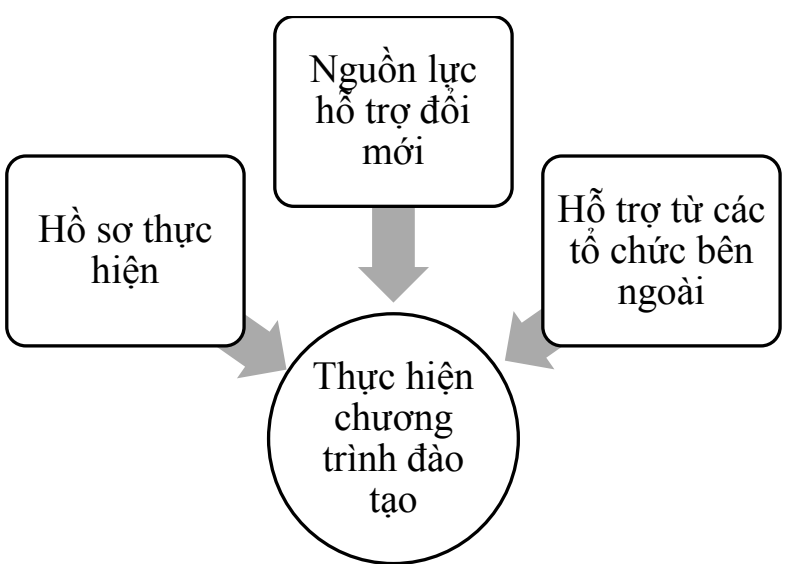

Nguồn: Rogan và Grayson, 2003

\section{Hình 1. Mô hình CIF}

\section{Hồ so' thực hiện (Profile of implementation)}

Xét về bản chất, hồ sơ thực hiện được xem như là một nỗ lực để hiểu và thể hiện mức độ lý tưởng mà các đề xuất chương trình giảng dạy đang được đưa vào thực tế. Theo đó, mỗi giáo viên sẽ có nhiều cách khác nhau để đưa một chương trình giảng dạy vào hoạt động. Hồ sơ thực hiện là điểm bắt đầu của quá trình cải cách chương trình giảng dạy, đóng vai trò như một bản đồ chỉ dẫn cho những người tham gia vào giai đoạn lập kế hoạch và thiết kế thay đổi chương trình giảng dạy, kết hợp các điểm mạnh và điểm yếu của tổ chức và con người trong tổ chức đó (Rogan và Grayson, 2003). Như vậy, cấu trúc này sẽ giải thích và so sánh các phương pháp khác nhau để áp dụng chương trình giảng dạy mới trong thực tiễn. Thông qua đó, cho phép các nhà hoạch định CTĐT xác định được vị trí và thế mạnh hiện tại của họ, kết hợp với bối cảnh và năng lực của tổ chức để lựa chọn một lộ trình nhằm xây dựng hoặc thay đổi chương trình hiệu quả trong một thời gian dài. Do đó, việc thực hiện một chương trình giảng dạy mới sẽ là một quá trình lâu dài, liên tục, trong đó giảng viên và các cá nhân khác cần nhận thức được điểm xuất phát và khả năng phát triển của mình.

Cách tiếp cận này rất phù hợp với khái niệm "lập kế hoạch phát triển" (Hargreaves và Hopkins, 1991), trong đó các thành viên khác nhau trong nhà trường tham gia xây dựng kế hoạch thực hiện thay đổi theo cách phù hợp và khả thi đối với bối cảnh và văn hóa của trường.

\section{Nguồn lực hỗ trợ đổi mới (Capacity to support innovation)}

Một khía cạnh quan trọng của quá trình thay đổi hay đổi mới là việc xem xét cách thức mà sự thay đổi/đổi mới có thể đạt được. Theo Feiter và cộng sự (1995), đối với các sáng kiến cải cách CTĐT, cần phải được thực hiện một cách tuần tự, có hệ thống bằng việc xem xét tất cả các yếu tố liên quan như điều kiện, sự đa dạng của các tổ chức, các vấn đề liên quan đến thực hiện và đảm bảo giám sát liên tục tiến độ trong và sau khi thực hiện. Tầm quan trọng của việc hiểu rõ Nguồn lực hỗ trợ đổi mới xuất phát từ sự khác biệt trong khả năng của các trường đại học khi thực hiện thay đổi một CTĐT và phải thừa nhận rằng không phải tất cả các trường học đều có khả năng thực hiện một sự thay đổi hay đổi mới ở cùng một mức độ như nhau. 
Nguồn lực hỗ trợ đổi mới được xem là một nỗ lực để hiểu và xây dựng các yếu tố có thể hỗ trợ hoặc cản trở việc thực hiện các ý tưởng và phương pháp mới trong một hệ thống, trong đó có trường học (Rogan và Grayson, 2003). Các khía cạnh để đánh giá và đo lường "Nguồn lực hỗ trợ đổi mới" của một tổ chức do Rogan và Grayson (2003) giới thiệu được phân loại theo bốn nhóm: (1) nguồn lực vật chất, (2) yếu tố liên quan đến giáo viên, (3) yếu tố liên quan đến người học và (4) môi trường và cơ chế quản lý của nhà trường.

Nguồn lự vật chất là yếu tố chính ảnh hưởng đến "Nguồn lực hỗ trợ đổi mới" của một tổ chức. Nguồn lực và điều kiện kém có thể hạn chế việc thực hiện một ý tưởng hay phương pháp mới (Howieson, 2003), làm suy yếu các nỗ lực tập trung học tập của người học, giới hạn khả năng của người dạy trong việc truyền đạt các khái niệm (Rogan và Grayson, 2003). Các yếu tố liên quan đến nguồn lực vật chất trong đào tạo có thể là tài liệu, giáo trình giảng dạy, cơ sở hạ tầng, thiết bị hỗ trợ và ngân hàng các tình huống (Ball và Cohen, 2000; Rogan và Grayson, 2003). Liên quan đến giảng dạy IFRS, việc thiếu tài nguyên giảng dạy có chất lượng cũng có thể là một trở ngại lớn đối với quá trình tích hợp IFRS vào CTĐT ở các trường đại học (Albader, 2015). Hiệp hội kế toán Hoa Kỳ và KPMG đã từng phối hợp thực hiện một khảo sát trên 535 khoa kế toán tại các trường đại học, kết quả là $62 \%$ khoa đào tạo đã không nỗ lực để tích hợp IFRS vào CTĐT của họ. Sự thiếu nỗ lực này có thể là do các giảng viên chưa sẵn sàng để đối đầu với các thay đổi, đặc biệt là nguồn lực hạn chế (tài liệu giảng dạy) do ngân sách eo hẹp (Singer, 2012).

Khía cạnh thứ hai liên quan đến giảng viên, bao gồm các yếu tố về đào tạo, mức độ tự tin của giảng viên và cam kết giảng dạy của họ. Jackling và cộng sự (2013) chỉ trích vai trò thụ động, không muốn thay đổi phương pháp sư phạm của các giảng viên kế toán tại Úc trước và sau khi áp dụng IFRS tại quốc gia này. Có thể thấy rõ, nền tảng kiến thức chuyên môn vững chắc sẽ ảnh hưởng lớn đến mức độ tự tin, cam kết thay đổi và sẵn sàng tham gia các hoạt động đào tạo của giảng viên. Bên cạnh đó, nhận thức của giảng viên về bản thân, quá trình nghiên cứu cũng như mức độ mà họ tin tưởng ở sự hỗ trợ đối với một sự đổi mới/ thay đổi trong nhà trường được chứng minh là đóng vai trò quan trọng, ảnh hưởng đến mức độ thích ứng của giáo viên với các thay đổi mới và việc sử dụng các giáo trình, tài liệu mới (Pintó và cộng sự, 2005).

Khía cạnh thứ ba ảnh hưởng đến việc thực hiện một CTĐT mới đó là người hocc. Nghiên cứu của Rogan và Grayson (2003) nhấn mạnh đến các vấn đề như gia đình, cam kết học tập, tính kỷ luật của người học và sự thành thạo trong ngôn ngữ giảng dạy. Trong một nghiên cứu của Bui và Porter (2010), thông qua khảo sát, nghiên cứu đã cho thấy tầm quan trọng của sự sẵn sàng để thay đổi phương pháp của sinh viên. Theo đó, sự nhiệt tình và chủ động để tiếp cận với phương pháp dạy và học mới của sinh viên sẽ có ảnh hưởng đáng kể đến mức độ sẵn sàng áp dụng các phương pháp giảng dạy mới của giảng viên.

Khía cạnh cuối cùng liên quan đến $m o ̂ i$ trường và cơ chế quản lý của tổ chức giáo dục. Một bộ máy quản lý xáo trộn, rối loạn chức năng sẽ là hạn chế lớn cho sự đổi mới/thay đổi CTĐT.

Một số nghiên cứu khác cũng đề cập đến các yếu tố ảnh hưởng tới sự đổi mới trong đào tạo kế toán. Howieson (2003) đã đưa ra bốn yếu tố đại diện cho các rào cản đối với bất kỳ sự thay đổi nào liên quan đến đào tạo kế toán, gồm: yếu tố đầu tiên liên quan đến mức độ nhận thức thấp của giảng viên và lãnh đạo nhà trường về ý nghĩa và sự cần thiết của sự thay đổi; yếu tố thứ hai là sự khan hiếm tài nguyên và vật chất, dẫn đến ít cơ hội cho phát triển và đổi mới; yếu tố thứ ba là sự thiếu nhận thức về chất lượng giảng dạy so với hoạt động nghiên cứu trong hệ thống của hầu hết các trường đại học, yếu tố thứ tư liên quan đến thời gian đào tạo, trong đó, các chương trình dài hạn (từ 3 đến 4 năm) có nhiều 
cơ hội đổi mới hơn các CTĐT ngắn hạn (từ 1 đến 2 năm).

\section{Hỗ trọ̣ từ các tổ chức bên ngoài (support from outside agencies)}

Các tổ chức bên ngoài được định nghĩa là các tổ chức bên ngoài trường, tương tác với trường học để tạo điều kiện cho việc thực hiện một CTĐT mới. Xây dựng, thực hiện một chương trình giảng dạy kết hợp các tài liệu và tài nguyên mới đòi hỏi phải có sự hỗ trợ cho các nhà giáo dục trong tất cả các giai đoạn của quá trình chuyển đổi sang IFRS (Penuel và cộng sự, 2007). Theo Porter và cộng sự (2003), các tổ chức bên ngoài có thể đóng góp một vai trò lớn trong việc cải thiện kiến thức của các nhà giáo dục kế toán về nội dung IFRS, cung cấp các tài liệu giảng dạy liên quan đến IFRS và nâng cao nhận thức của các học giả về các phương pháp và kỹ thuật sư phạm phù hợp nhất với IFRS. IFAC (2006) kỳ vọng rằng các hiệp hội nghề nghiệp sẽ hỗ trợ quá trình áp dụng
IFRS thông qua vai trò của họ trong việc cung cấp các CTĐT và các khóa cập nhật kiến thức chuyên môn thường xuyên (CPD - Continuing Professional Development).

Nghiên cứu Smylie (1997), Ball và Cohen (1996), Curuk và Dogan (2002), Yildiz và Durak (2011), Kutluk và cộng sự (2012) cho rằng để tăng hiệu quả của phát triển chuyên môn trong quá trình thực hiện chương trình giảng dạy mới, các hoạt động phát triển chuyên môn phù hợp liên quan đến chương trình giảng dạy mới cần được xác định. Quá trình chuyển đổi sang IFRS tại các quốc gia sẽ tạo ra nhu cầu đào tạo lớn, vì vậy vai trò của các hiệp hội nghề nghiệp và các tổ chức học thuật bên ngoài cần phải được nghiên cứu và đánh giá. Ngoài ra, kết các nghiên cứu cũng cho kết quả rằng chương trình học không đáp ứng đầy đủ nhu cầu của doanh nghiệp, do đó sinh viên cần được tiếp xúc với thực tế nhiều hơn, tăng cơ hội tham quan, giao lưu và học hỏi từ các doanh nghiệp.

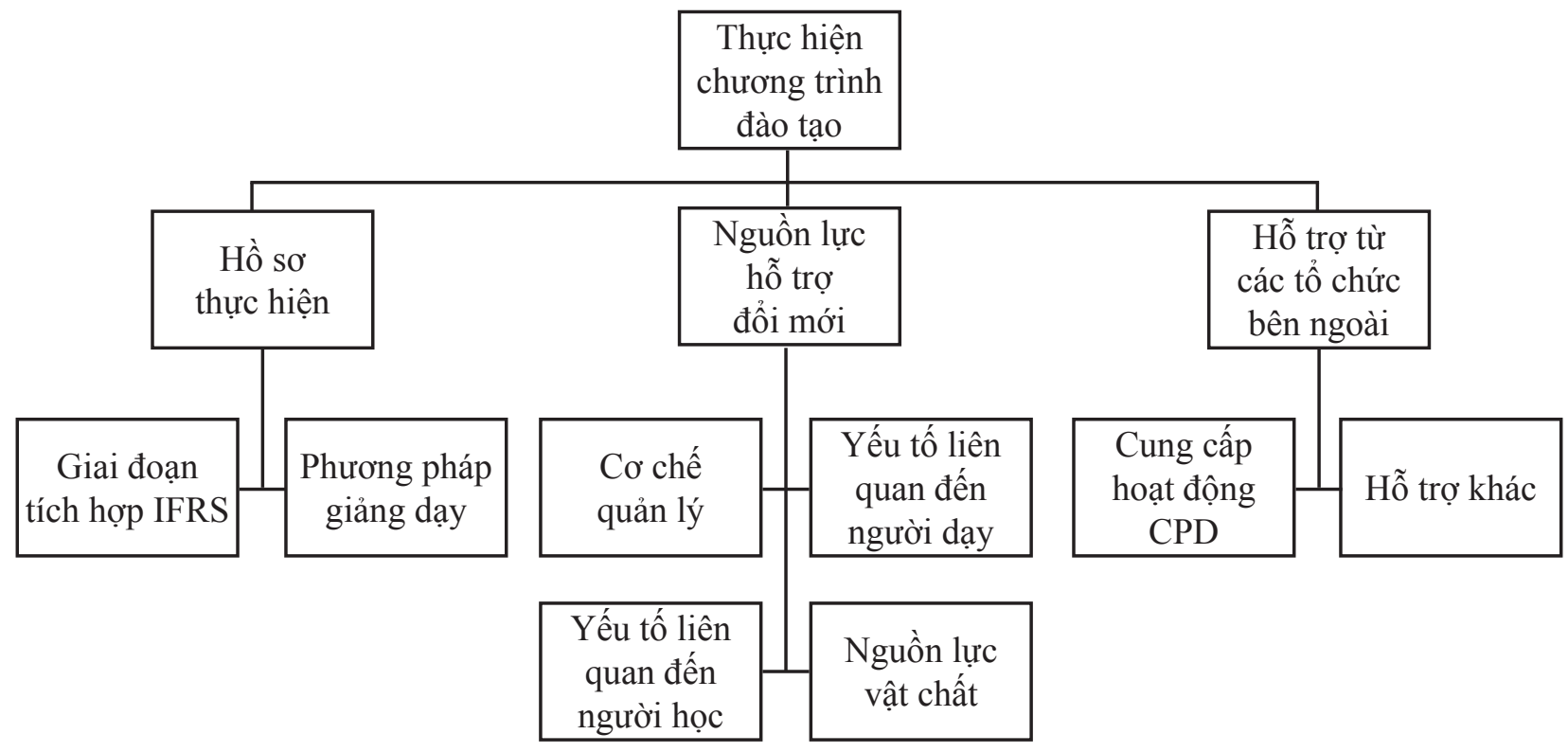

Nguồn: Tổng hợp của nhóm tác giả

Hình 2. Mô hình CIF mở rộng

Từ mô hình CIF cho thấy, các yếu tố ảnh hưởng gồm: yếu tố giai đoạn tích hợp IFRS, yếu tố phương pháp giảng dạy, yếu tố nhận thức về rào cản (yếu tố nguồn lực vật chất, yếu tố liên quan đến người dạy, yếu tố liên quan đến người học, yếu tố cơ chế quản lý), yếu tố hỗ trợ từ các tổ chức bên ngoài ảnh hưởng đến quá trình tích hợp IFRS vào CTĐT chuyên ngành kế toán, kiểm toán. 


\section{Phương pháp nghiên cứu}

\subsection{Phương pháp nghiên cúu đự̣c sử dụng}

Nghiên cứu chủ yếu sử dụng phương pháp phỏng vấn bằng bảng câu hỏi và phương pháp tổng hợp thống kê mô tả. Câu hỏi phục vụ thu thập dữ liệu được xây dựng và phát triển từ cơ sở lý thuyết nền CIF của Rogan và Grayson (2003). Đặc biệt, nhóm tác giả dựa vào thang đo được sử dụng trong khảo sát của KPMG/AAA IFRS Survey (2011) và Albader (2015). Trong đó, các câu hỏi liên quan đến cấu trúc Hồ sơ năng lực thực hiện và Nguồn lực hỗ trợ đổi mới chủ yếu được xây dựng dựa trên bảng khảo sát của KPMG/AAA (2011). Câu hỏi trong bảng hỏi được thiết kế dưới nhiều dạng như: câu hỏi mở, câu hỏi có hai sự lựa chọn, câu hỏi nhiều sự lựa chọn, danh sách, xếp hạng và thang đo likert.

\subsection{Mẫu nghiên cứu và phương pháp thu thập dĩ liệu}

Bảng câu hỏi khảo sát được gởi đến các giảng viên và sinh viên khoa Kế toán - Kiểm toán, trường $\mathrm{ĐH}$ Tài chính - Marketing bằng bản giấy và bản online. Kết quả, nhóm tác giả thu về được 180 bảng trả lời câu hỏi, trong đó bao gồm 32 giảng viên và 148 sinh viên các khóa. Sau khi tiến hành sàng lọc và loại bỏ các phiếu khảo sát không đạt yêu cầu, thiếu thông tin hoặc trả lời thiếu khách quan, còn lại 174 phiếu đạt yêu cầu (đạt tỷ lệ 96,67\%). Trong số 174 đối tượng khảo sát, trong đó có 32 giảng viên và 142 sinh viên khoa Kế toán - Kiểm toán, UFM, tập trung vào sinh viên từ năm 2 đến năm $4(83,8 \%)$ và sinh viên đã tốt nghiệp $(16,2 \%)$.

\subsection{Phương pháp xủ̉ lý và phân tích dũ liệu}

Nhóm tác giả tiến hành tập hợp dữ liệu từ bảng trả lời câu hỏi, và sử dụng phần mềm SPSS, phần mềm excel để hỗ trợ phân tích dữ liệu. Sử dụng phương pháp thống kê mô tả dữ liệu, tính toán số trung bình, tỷ lệ, tần suất, độ lệch chuẩn. Từ đó, đánh giá quá trình tích hợp IFRS trong CTĐT chuyên ngành kế toán, kiểm toán của UFM.

\section{Kết quả nghiên cứu}

\subsection{Kết quả nghiên cúu yếu tố giai đọ̣n tích hoop IFRS}

Đối với yếu tố “Giai đoạn tích hợp”, nhóm tác giả thực hiện khảo sát giảng viên và sinh viên nhằm đánh giá nhận thức của họ về tầm quan trọng, thời điểm kết hợp IFRS vào chương trình giảng dạy kế toán, kiểm toán, và trình độ học vấn cần được tích hợp. Từ đó, xác định giai đoạn tích hợp IFRS vào CTĐT của trường $Đ H$ Tài chính - Marketing.

Bảng 1. Nhận thức của giảng viên và sinh viên về tầm quan trọng của việc tích hợp IFRS và lộ trình áp dụng IFRS

\begin{tabular}{|c|c|c|c|c|}
\hline & \multicolumn{2}{|c|}{$\begin{array}{l}\text { Tầm quan trọng của việc tích hợp } \\
\text { IFRS vào CTĐT }\end{array}$} & \multicolumn{2}{|c|}{$\begin{array}{l}\text { Nhận định Việt Nam thực hiện đúng } \\
\text { lộ trình áp dụng IFRS vào năm } 2020\end{array}$} \\
\hline & Số lần & Tỷ lệ (\%) & Số lần & Tỷ lệ (\%) \\
\hline Rất không đồng ý & 2 & 1,1 & 5 & 2,9 \\
\hline Không đồng ý & 0 & 0,0 & 19 & 10,9 \\
\hline Không ý kiến & 11 & 6,3 & 52 & 29,9 \\
\hline Đồng ý & 103 & 59,2 & 87 & 50,0 \\
\hline Rất đồng ý & 58 & 33,3 & 11 & 6,3 \\
\hline Total & 174 & 100,0 & 174 & 100,0 \\
\hline Mean & \multicolumn{2}{|r|}{4,24} & \multicolumn{2}{|r|}{3,46} \\
\hline Std. Deviation & \multicolumn{2}{|r|}{0,669} & \multicolumn{2}{|r|}{0,878} \\
\hline
\end{tabular}

Nguồn: Kết quả khảo sát của nhóm tác giả 
Kết quả thống kê Bảng 1 cho thấy, phần lớn giảng viên và sinh viên đều nhận thấy được sự cần thiết của việc giảng dạy IFRS (mean = 4,24). Cụ thể, $92,5 \%$ đối tượng khảo sát cho rằng việc tích hợp IFRS vào $\mathrm{CTĐT} \mathrm{là} \mathrm{quan}$ trọng và rất quan trọng, cho thấy sự sẵn sàng và chấp nhận của các giảng viên và sinh viên đối với một sự thay đổi, đổi mới trong CTĐT. Nhận thức về mức độ quan trọng của việc tích hợp IFRS vào giảng dạy được kỳ vọng sẽ ảnh hưởng tích cực tới tiến độ của quá trình tích hợp và mức độ mà IFRS được tích hợp vào $\mathrm{CTĐT}$ của trường. 56,3\% kết quả khảo sát cho rằng Việt Nam sẽ thực hiện đúng lộ trình áp dụng IFRS vào năm 2020. Điều này thể hiện được tính cần thiết của việc giảng dạy IFRS cho sinh viên đại học, cũng như thời điểm tích hợp cần phải được xem xét kỹ để giúp sinh viên có được những kiến thức, kỹ năng cơ bản của IFRS, đáp ứng nhu cầu lao động thực tế trong tương lai.

Bảng 2. Mức độ yêu thích học IFRS của sinh viên

\begin{tabular}{|l|c|c|c|c|c|}
\hline \multicolumn{1}{|c|}{ Mức độ } & Số lần & Tỷ lệ (\%) & Mức độ & Số lần & Tỷ lệ (\%) \\
\hline Rất không thích & 1 & .7 & Thích & 72 & 50.7 \\
\hline Không thích & 3 & 2.1 & Rất thích & 12 & 8.5 \\
\hline Không ý kiến & 54 & 38.0 & Total & $\mathbf{1 4 2}$ & $\mathbf{1 0 0 . 0}$ \\
\hline $\begin{array}{l}\text { Mean = 3.64, Std. Deviation =.698, } \\
\text { Min =1, Max =5 }\end{array}$ & & & & \\
\hline
\end{tabular}

Nguồn: Kết quả khảo sát của nhóm tác giả

Kết quả Bảng 2 cho thấy $59,2 \%$ sinh viên thích và rất thích được học IFRS trên giảng đường đại học và sinh viên nhận thấy lợi ích mà IFRS mang lại, trong đó, lý do thúc đẩy việc tìm hiểu IFRS lớn nhất là giúp sinh viên có được công việc tốt trong tương lai (mean =
4.24), giúp họ có khả năng xử lý, hạch toán các nghiệp vụ giao dịch quốc tế (mean = 4.13) (kết quả bảng 3 ). Điều này cho thấy, nếu quá trình thay đổi CTĐT theo hướng tích hợp IFRS diễn ra, sẽ nhận được sự ủng hộ tích cực từ chính chủ thể của quá trình đó.

Bảng 3. Lý do thích học IFRS của sinh viên

\begin{tabular}{|l|c|c|c|}
\hline & $\mathrm{N}$ & Mean & Std. Deviation \\
\hline IFRS sẽ được áp dụng tại Việt Nam trong thời gian tới & 84 & 3.75 & .876 \\
\hline Giúp tôi cập nhật kiến thức chuyên ngành & 84 & 4.04 & .768 \\
\hline $\begin{array}{l}\text { Giúp tôi chuẩn bị kiến thức để tham dự các cuộc thi lấy chứng chì' } \\
\text { học bống liên quan tới kế toán, kiểm toán }\end{array}$ & 84 & 4.02 & .891 \\
\hline Giúp tôi có được công việc tốt & 84 & 4.24 & .873 \\
\hline Giúp tôi có khả năng xử lý, hạch toán các nghiệp vụ giao dịch quốc tế & 84 & 4.13 & .847 \\
\hline
\end{tabular}

Nguồn: Kết quả khảo sát của nhóm tác giả

Bảng 4. Mong muốn về thời điểm nên tích hợp IFRS vào CTĐT

\begin{tabular}{|l|c|c|c|c|c|c|}
\hline \multirow{2}{*}{ Nội dung } & \multicolumn{2}{|c|}{ Giảng viên } & \multicolumn{2}{c|}{ Sinh viên } & \multicolumn{2}{c|}{ Tổng cộng } \\
\cline { 2 - 8 } & SL & Tỷ lệ & SL & Tỷ lệ & SL & Tỳ lệ \\
\hline $\begin{array}{l}\text { Tôi không tin là IFRS sẽ được tích hợp đáng kề } \\
\text { vào CTĐT }\end{array}$ & 3 & 9,38 & 10 & 7,04 & 13 & 7,47 \\
\hline Bắt đầu từ năm học 2021 -2022 & 17 & 53,13 & 31 & 21,83 & 48 & 27,59 \\
\hline Bắt đầu từ năm học 2020 -2021 & 8 & 25,00 & 64 & 45,07 & 72 & 41,38 \\
\hline Bắt đầu từ năm học 2019 -2020 & 1 & 3,13 & 28 & 19,72 & 29 & 16,67 \\
\hline IFRS đã được tích hợp vào CTĐT hiện hành & 3 & 9,38 & 9 & 6,34 & 12 & 6,90 \\
\hline \multicolumn{1}{|c|}{ Tồng cộng } & $\mathbf{3 2}$ & $\mathbf{1 0 0}$ & $\mathbf{1 4 2}$ & $\mathbf{1 0 0}$ & $\mathbf{1 7 4}$ & $\mathbf{1 0 0}$ \\
\hline
\end{tabular}

Nguồn: Kết quả khảo sát của nhóm tác giả 
Kết quả khảo sát ở Bảng 4 cho thấy, chỉ có $7,47 \%$ giảng viên và sinh viên không tin IFRS sẽ được tích hợp đáng kể vào CTĐT và $27,59 \%$ số người được hỏi cho rằng IFRS sẽ không được tích hợp trước năm 2021. Kết quả này còn cho thấy sự khác biệt về thời điểm mong muốn tích hợp IFRS trong giảng dạy giữa hai đối tượng.
$53,13 \%$ giảng viên mong muốn IFRS chỉ nên được tích hợp đáng kể vào chương trình giảng dạy cho khóa sinh viên từ năm 2021 trở đi. Trong khi đó, 45,07\% sinh viên lại mong muốn được tích lũy đầy đủ các kiến thức cơ bản về IFRS bắt đầu từ năm 2020 .

\section{Bảng 5. Mong muốn cấp độ đào tạo được tích hợp IFRS}

\begin{tabular}{|c|c|c|c|c|c|c|}
\hline \multirow{2}{*}{ Nội dung } & \multicolumn{2}{|c|}{ Giảng viên } & \multicolumn{2}{c|}{ Sinh viên } & \multicolumn{2}{c|}{ Tổng cộng } \\
\cline { 2 - 7 } & SL & Tỷ lệ & SL & Tỷ lệ & SL & Tỷ lệ \\
\hline Đại học & 8 & $25 \%$ & 24 & $16,9 \%$ & 32 & $18,39 \%$ \\
\hline Sau đại học & 2 & $6,25 \%$ & 33 & $23,24 \%$ & 35 & $20,11 \%$ \\
\hline Cả 2 & 22 & $68,75 \%$ & 85 & $59,86 \%$ & 107 & $61,49 \%$ \\
\hline Tổng cộng & $\mathbf{3 2}$ & $\mathbf{1 0 0 \%}$ & $\mathbf{1 4 2}$ & $\mathbf{1 0 0}$ & $\mathbf{1 7 4}$ & $\mathbf{1 0 0}$ \\
\hline
\end{tabular}

Nguồn: Kết quả khảo sát của nhóm tác giả

Kết quả Bảng 5 cho thấy, 61,49\% đối tượng khảo sát cho rằng IFRS nên được tích hợp vào CTĐT ở cả hai bậc đào tạo: đại học và sau đại học. Kết quả này đưa đến một gợi ý cho trường
ĐH Tài chính - Marketing, đó là nên thực hiện từng bước để tích hợp IFRS vào CTĐT đại học hiện tại và bậc đào tạo cao hơn trong tương lai (Cao học).

\section{Bảng 6. Nhận định công tác chuẩn bị tích hợp IFRS tại đơn vị đào tạo}

\begin{tabular}{|l|c|c|}
\hline \multicolumn{1}{|c|}{ Nội dung } & \multicolumn{2}{c|}{ Giảng viên } \\
\cline { 2 - 3 } & SL & Tỷ lệ \\
\hline Chưa triển khai chuẩn bị & 3 & $9,38 \%$ \\
\hline Chủ động đánh giá, xem xét việc tích hợp cho các khóa học trong tương lai & 15 & $46,88 \%$ \\
\hline Đã xây dựng một môn học riêng về IFRS & 3 & $9,38 \%$ \\
\hline Đã tích hợp các nội dung quan trọng của IFRS vào một số môn học hiện hành & 11 & $34,38 \%$ \\
\hline Tổng cộng & $\mathbf{3 2}$ & $\mathbf{1 0 0 \%}$ \\
\hline
\end{tabular}

Nguồn: Kết quả khảo sát của nhóm tác giả

Kết quả Bảng 6 cho thấy 34,38\% giảng viên cho rằng IFRS đã được tích hợp một số nội dung quan trọng vào một số môn học hiện hành như khái niệm và chuẩn mực kế toán, kế toán quốc tế, lập BCTC quốc tế...

\section{Bảng 7. Mong đợi về khóa sinh viên tốt nghiệp sẽ có đầy đủ kiến thức về IFRS}

\begin{tabular}{|c|c|c|c|c|c|c|}
\hline \multirow{2}{*}{ Nội dung } & \multicolumn{2}{|c|}{ Giảng viên } & \multicolumn{2}{c|}{ Sinh viên } & \multicolumn{2}{c|}{ Tổng cộng } \\
\cline { 2 - 7 } & SL & Tỷ lệ & SL & Tỷ lệ & SL & Tỷ lệ \\
\hline Các khóa nhập học từ 2021 trở đi & 17 & 53,13 & 40 & 28,17 & 57 & 32,76 \\
\hline Khóa 2020-2024 & 12 & 37,50 & 32 & 22,54 & 44 & 25,29 \\
\hline Khóa 2019-2023 & 1 & 3,13 & 24 & 16,90 & 25 & 14,37 \\
\hline Khóa 2018-2022 & 1 & 3,13 & 18 & 12,68 & 19 & 10,92 \\
\hline Khóa 2017-2021 & 1 & 3,13 & 28 & 19,72 & 29 & 16,67 \\
\hline Tổng cộng & $\mathbf{3 2}$ & $\mathbf{1 0 0}$ & $\mathbf{1 4 2}$ & $\mathbf{1 0 0}$ & $\mathbf{1 7 4}$ & $\mathbf{1 0 0}$ \\
\hline
\end{tabular}

Nguồn: Kết quả khảo sát của nhóm tác giả 
Đa số giảng viên và sinh viên $(32,76 \%)$ tin rằng sẽ không có sinh viên tốt nghiệp với đầy đủ kiến thức về IFRS tham gia vào thị trường lao động cho đến ít nhất là năm 2025 (khóa sinh viên nhập học từ năm 2021 trở đi).

\subsection{Kết quả nghiên cứu yếu tố phương pháp giảng dạy}

Bảng 8 cho thấy, hướng tiếp cận được đánh giá cao nhất là tiếp tục giảng dạy chuẩn mực Việt Nam và giới thiệu IFRS thông qua so sánh và đối chiếu khung khái niệm của IFRS với chuẩn mực Việt Nam (principle-based) (mean $=3.80)$.

Bảng 8. Hướng tiếp cận phù hợp khi giảng IFRS và Kiểm định Mann-Whitney Test

\begin{tabular}{|c|c|c|c|c|c|c|c|c|}
\hline \multirow{2}{*}{\multicolumn{2}{|c|}{$\begin{array}{c}\text { Hướng tiếp cận phù hợp khi giảng } \\
\text { dạy IFRS }\end{array}$}} & \multirow[b]{2}{*}{$\mathrm{N}$} & \multirow[b]{2}{*}{ Mean } & \multirow{2}{*}{$\begin{array}{c}\text { Std. } \\
\text { Deviation } \\
\text { Đối } \\
\text { tượng }\end{array}$} & \multicolumn{4}{|c|}{ Kiểm định Mann-Whitney Test } \\
\hline & & & & & $\mathrm{N}$ & Mean & Sum of & \\
\hline \multirow{2}{*}{$\begin{array}{l}\text { Hướng } \\
\text { tiếp cận } \\
1\end{array}$} & \multirow{2}{*}{$\begin{array}{l}\text { Tiếp tục giảng dạy chuẩn } \\
\text { mực Việt Nam và sẽ giới } \\
\text { thiệu IFRS thông qua so } \\
\text { sánh và đối chiếu khung khái } \\
\text { niệm của IFRS với chuẩn } \\
\text { mực Việt Nam (principle- } \\
\text { based) }\end{array}$} & \multirow[b]{2}{*}{174} & \multirow[b]{2}{*}{3,80} & \multirow[b]{2}{*}{0,873} & Giảng viên & 32 & 95.67 & 3061.5 \\
\hline & & & & & Sinh viên & 142 & 85.66 & 12163.5 \\
\hline \multirow{2}{*}{$\begin{array}{l}\text { Hướng } \\
\text { tiếp cận } \\
2\end{array}$} & \multirow{2}{*}{$\begin{array}{l}\text { Tiếp tục giảng dạy chuẩn } \\
\text { mực Việt Nam và sẽ giới } \\
\text { thiệu IFRS thông qua so sánh } \\
\text { và đối chiếu các kỹ thuật của } \\
\text { IFRS với chuẩn mực Việt } \\
\text { Nam (rule-based) }\end{array}$} & \multirow[b]{2}{*}{174} & \multirow[b]{2}{*}{3,78} & \multirow[b]{2}{*}{0,825} & Giảng viên & 32 & 89.56 & 2866 \\
\hline & & & & & Sinh viên & 142 & 87.04 & 12359 \\
\hline \multirow{2}{*}{$\begin{array}{l}\text { Hướng } \\
\text { tiếp cận } \\
3\end{array}$} & \multirow{2}{*}{$\begin{array}{l}\text { Tiếp tục giảng dạy chuẩn } \\
\text { mực Việt Nam và chỉ đề cập } \\
\text { đến IFRS một phần trong đó }\end{array}$} & \multirow[b]{2}{*}{174} & \multirow[b]{2}{*}{3,01} & \multirow[b]{2}{*}{1,112} & Giảng viên & 32 & 86.59 & 2771 \\
\hline & & & & & Sinh viên & 142 & 87.70 & 12454 \\
\hline \multirow{2}{*}{$\begin{array}{l}\text { Hướng } \\
\text { tiếp cận } \\
4\end{array}$} & \multirow{2}{*}{$\begin{array}{l}\text { Chỉ giảng dạy về khái niệm } \\
\text { và nguyên tắc của IFRS }\end{array}$} & \multirow[b]{2}{*}{174} & \multirow[b]{2}{*}{2,73} & \multirow[b]{2}{*}{1,144} & Giảng viên & 32 & 80.62 & 2580 \\
\hline & & & & & Sinh viên & 142 & 89.05 & 12645 \\
\hline
\end{tabular}

\begin{tabular}{|l|r|r|r|r|}
\hline \multicolumn{7}{|c|}{ Test Statistics $^{\mathbf{a}}$} \\
\hline & $\begin{array}{c}\text { Hướng tiếp cận } \\
1\end{array}$ & $\begin{array}{c}\text { Hướng tiếp cận } \\
2\end{array}$ & $\begin{array}{c}\text { Hướng tiếp cận } \\
3\end{array}$ & $\begin{array}{c}\text { Hướng tiếp cận } \\
4\end{array}$ \\
\hline Mann-Whitney U & 2010,500 & 2206,000 & 2243,000 & 2052,000 \\
\hline Wilcoxon W & 12163,500 & 12359,000 & 2771,000 & 2580,000 \\
\hline Z & $-1,108$ &,- 281 &,- 117 &,- 882 \\
\hline Asymp. Sig. (2-tailed) &, 268 &, 778 &, 907 &, 378 \\
\hline a. Grouping Variable: Doi tuong & \multicolumn{3}{|l}{} \\
\hline
\end{tabular}


Khi thực hiện kiểm định Mann-Whitney Test, với giá trị p-value lần lượt là $0,268,0,778$, 0,907 và $0,378(>0.05)$, có thể kết luận trung bình đánh giá về hướng tiếp cận khi giảng dạy IFRS giữa giảng viên và sinh viên là như nhau tại mức ý nghĩa 5\%.

Bảng 9. Đánh giá về mức độ hữu ích của các phương pháp giảng dạy

\begin{tabular}{|c|l|c|c|c|}
\hline Mã & \multicolumn{1}{|c|}{ Nội dung } & $\mathrm{N}$ & Mean & $\begin{array}{c}\text { Std. } \\
\text { Deviation }\end{array}$ \\
\hline PP1 & $\begin{array}{l}\text { Khách mời (chuyên gia về IFRS) sẽ cung cấp kinh nghiệm } \\
\text { thực tế cho sinh viên }\end{array}$ & 174 & 4.18 & .887 \\
\hline PP2 & Giới thiệu các công trình nghiên cứu liên quan đến IFRS & 174 & 3.75 & .926 \\
\hline PP3 & Thực hành, mô phỏng (các vấn đề kinh doanh thực tế) & 174 & 4.32 & .759 \\
\hline PP4 & Phương pháp học qua vấn đề (Problem - based Learning) & 174 & 4.22 & .782 \\
\hline PP5 & $\begin{array}{l}\text { Sử dụng case studies nhằm phát triển khả năng tư duy, kỹ } \\
\text { năng phân tích, đánh giá của sinh viên }\end{array}$ & 174 & 4.27 & .754 \\
\hline PP6 & So sánh song song IFRS và chuẩn mực kế toán Việt Nam & 174 & 4.11 & .829 \\
\hline PP7 & $\begin{array}{l}\text { Giảng dạy khuôn mẫu lý thuyết, các khái niệm của IFRS } \\
\text { trước khi đi vào các nguyên tắc }\end{array}$ & 174 & 3.85 & .925 \\
\hline PP8 & $\begin{array}{l}\text { Sinh viên tìm hiểu và thuyết trình về các chủ đề liên quan } \\
\text { đến IFRS }\end{array}$ & 174 & 3.75 & 1.017 \\
\hline
\end{tabular}

Nguồn: Kết quả khảo sát của nhóm tác giả

Các đối tượng khảo sát đã thể hiện quan điểm của họ đối với các phương pháp giảng dạy IFRS thông qua đánh giá tính hữu ích của phương pháp đó theo thang đo Likert 5 mức độ. Kết quả khảo sát cho thấy có $5 / 8$ phương pháp được đánh giá có tính hữu ích cao đối với việc giảng dạy IFRS (mean > 4.0), bao gồm: thực hành, mô phỏng (mean $=4,32)$; sử dụng phương pháp tình huống (mean $=4,27$ ); phương pháp học qua vấn đề (mean $=4,22)$; khách mời, chuyên gia IFRS (mean $=4,18)$ và so sánh IFRS và chuẩn mực kế toán hiện hành $($ mean $=4,11)$. Các phương pháp như thuyết trình, giới thiệu các công trình nghiên cứu về IFRS và giảng dạy khuôn mẫu lý thuyết không được xem xét là có giá trị cao như các phương pháp khác. Từ đó có thể thấy được nhận thức của các đối tượng khảo sát về giá trị của các phương pháp giảng dạy lấy người học làm trung tâm, phản ánh được sự sẵn sàng của các đối tượng về việc sử dụng các phương pháp đó trong tương lai.

\section{Bảng 10. Kiểm định Mann-Whitney Test}

\begin{tabular}{|l|c|c|c|c|c|c|c|c|c|}
\hline & & \multicolumn{2}{|c|}{ PP1 } & \multicolumn{2}{c|}{ PP2 } & \multicolumn{2}{c|}{ PP3 } & \multicolumn{2}{c|}{ PP4 } \\
\hline Đối tượng & $\mathrm{N}$ & $\begin{array}{c}\text { Mean } \\
\text { Rank }\end{array}$ & $\begin{array}{c}\text { Sum of } \\
\text { Ranks }\end{array}$ & $\begin{array}{c}\text { Mean } \\
\text { Rank }\end{array}$ & $\begin{array}{c}\text { Sum of } \\
\text { Ranks }\end{array}$ & $\begin{array}{c}\text { Mean } \\
\text { Rank }\end{array}$ & $\begin{array}{c}\text { Sum of } \\
\text { Ranks }\end{array}$ & $\begin{array}{c}\text { Mean } \\
\text { Rank }\end{array}$ & $\begin{array}{c}\text { Sum of } \\
\text { Ranks }\end{array}$ \\
\hline Giảng viên & 32 & 96,14 & 3076,50 & 78,08 & 2498,50 & 93,19 & 2982 & 93,38 & 2988 \\
\hline Sinh viên & 142 & 85,55 & 12148,50 & 89,62 & 12726,50 & 86,22 & 12243 & 86,18 & 12237 \\
\hline
\end{tabular}

\begin{tabular}{|l|c|c|c|c|c|c|c|c|c|}
\hline & & \multicolumn{2}{|c|}{ PP5 } & \multicolumn{2}{c|}{ PP6 } & \multicolumn{2}{c|}{ PP7 } & \multicolumn{2}{c|}{ PP8 } \\
\hline Đối tượng & $\mathrm{N}$ & $\begin{array}{c}\text { Mean } \\
\text { Rank }\end{array}$ & $\begin{array}{c}\text { Sum of } \\
\text { Ranks }\end{array}$ & $\begin{array}{c}\text { Mean } \\
\text { Rank }\end{array}$ & $\begin{array}{c}\text { Sum of } \\
\text { Ranks }\end{array}$ & $\begin{array}{c}\text { Mean } \\
\text { Rank }\end{array}$ & $\begin{array}{c}\text { Sum of } \\
\text { Ranks }\end{array}$ & $\begin{array}{c}\text { Mean } \\
\text { Rank }\end{array}$ & $\begin{array}{c}\text { Sum of } \\
\text { Ranks }\end{array}$ \\
\hline Giảng viên & 32 & 96,14 & 3076,5 & 90,44 & 2894 & 107,81 & 3450 & 98,62 & 3156 \\
\hline Sinh viên & 142 & 85,55 & 12148,5 & 86,84 & 12331 & 82,92 & 11775 & 84,99 & 12069 \\
\hline
\end{tabular}




\begin{tabular}{|l|r|r|r|r|r|r|r|r|}
\hline \multicolumn{7}{|c|}{ Test Statistics $^{\mathbf{a}}$} \\
\hline & \multicolumn{1}{|c|}{ PP1 } & \multicolumn{1}{c|}{ PP2 } & \multicolumn{1}{|c|}{ PP3 } & \multicolumn{1}{c|}{ PP4 } & \multicolumn{1}{c|}{ PP5 } & \multicolumn{1}{c|}{ PP6 } & \multicolumn{1}{c|}{ PP7 } & \multicolumn{1}{c|}{ PP8 } \\
\hline Mann-Whitney U & 1995,5 & 1970,5 & 2090 & 2084 & 1599,5 & 2178 & 1622 & 1916 \\
\hline Wilcoxon W & 12148,5 & 2498,5 & 12243 & 12237 & 11752,5 & 12331 & 11775 & 12069 \\
\hline Z & $-1,154$ & $-1,241$ &,- 776 &,- 791 & $-2,859$ &,- 393 & $-2,673$ & $-1,459$ \\
\hline Asymp. Sig. (2-tailed) &, 249 &, 214 &, 438 &, 429 &, 004 &, 694 &, 008 &, 145 \\
\hline \multicolumn{2}{|l|}{} \\
a. Grouping Variable: Doi tuong & & & & & & \\
\hline
\end{tabular}

Nguồn: Kết quả khảo sát của nhóm tác giả

Kết quả kiểm định Mann-Whitney Test cho thấy có sự khác biệt trung bình đánh giá phương pháp giảng dạy IFRS giữa sinh viên và giảng viên tại nhóm phương pháp số 5 và 7 (p-value < 0.05). Cụ thể, sử dụng tình huống và giảng dạy khuôn mẫu lý thuyết, các khái niệm của IFRS trước khi đi vào các nguyên tắc là 2 phương pháp được đối tượng giảng viên đánh giá cao hơn sinh viên (mean rank lần lượt là 108,62 (> $82,76)$ và $107,81(82,92)$.

\section{Bảng 11. Nội dung các tình huống dùng trong giảng dạy IFRS}

\begin{tabular}{|l|c|c|c|c|c|c|}
\hline \multicolumn{1}{|c|}{ Nội dung } & \multicolumn{2}{c|}{ Giảng viên } & \multicolumn{2}{|c|}{ Sinh viên } & \multicolumn{2}{c|}{ Tổng cộng } \\
\cline { 2 - 9 } & SL & Tỷ lệ & SL & Tỷ lệ & SL & Tỷ lệ \\
\hline $\begin{array}{l}\text { Các tình huống nên tập trung vào phương diện kỹ } \\
\text { thuật của IFRS (tiếp cận theo hướng quy tắc - rule } \\
\text { based) }\end{array}$ & 4 & 12,50 & 16 & 11,27 & 20 & 11,49 \\
\hline $\begin{array}{l}\text { Các tình huống nên tập trung vào các khái niệm nền } \\
\text { tảng của IFRS (tiếp cận theo hướng nguyên tắc }- \\
\text { principle based) }\end{array}$ & 2 & 6,25 & 10 & 7,04 & 12 & 6,90 \\
\hline Cả hai nội dung trên & 26 & 81,25 & 116 & 81,69 & 142 & 81,61 \\
\hline Tổng cộng & 32 & 100 & 142 & 100 & 174 & 100 \\
\hline
\end{tabular}

Nguồn: Kết quả khảo sát của nhóm tác giả

4.3. Kết quả nghiên cứu yếu tố nhận thức $\quad$ kế hoạch chuẩn bị của lãnh đạo đơn vị đối với rào cản quá trình tích hợp IFRS

Nhận định của giảng viên về sự hiểu biết và

Bảng 12. Đánh giá yếu tố cơ chế quản lý

\begin{tabular}{|l|c|c|c|c|c|}
\hline $\begin{array}{l}\text { 1. Mức độ hiểu biết về nhu cầu phải } \\
\text { thay đổi CTĐT để đáp ứng quá trình } \\
\text { chuyển đổi IFRS của lãnh đạo đơn } \\
\text { vị }\end{array}$ & SL & Tỷ lệ & $\begin{array}{l}\text { 2. Lãnh đạo đơn vị công } \\
\text { tác đã có kế hoạch gì để } \\
\text { đào tạo giảng viên giảng } \\
\text { dạy IFRS (được lựa chọn } \\
\text { nhiều phương án) }\end{array}$ & Tỷ lệ \\
\hline Rất thấp & 1 & 3,13 & $\begin{array}{l}\text { Phân công, chỉ định giảng } \\
\text { viên giảng dạy IFRS }\end{array}$ & 13 & 40,63 \\
\hline Thấp & 2 & 6,25 & $\begin{array}{l}\text { Có kế hoạch hỗ trợ kinh } \\
\text { phí đào tạo cho giảng viên } \\
\text { hiện tại }\end{array}$ & 14 & 43,75 \\
\hline
\end{tabular}




\begin{tabular}{|l|c|c|l|c|c|}
\hline $\begin{array}{l}\text { 1. Mức độ hiểu biết về nhu cầu phải } \\
\text { thay đổi CTĐT để đáp ứng quá trình } \\
\text { chuyển đổi IFRS của lãnh đạo đơn } \\
\text { vị }\end{array}$ & SL & Tỷ lệ & $\begin{array}{l}\text { 2. Lãnh đạo đơn vị công } \\
\text { tác đã có kế hoạch gì để } \\
\text { đào tạo giảng viên giảng } \\
\text { dạy IFRS (đuợc lụa chọn } \\
\text { nhiều phuóng án) }\end{array}$ & Tỷ lệ \\
\hline Trung bình & 13 & 40,63 & $\begin{array}{l}\text { Có kế hoạch hỗ trợ kinh } \\
\text { phí cho việc biên soạn tài } \\
\text { liệu giảng dạy }\end{array}$ & 5 & 15,63 \\
\hline Cao & 9 & 28,13 & $\begin{array}{l}\text { Mời giảng viên giảng các } \\
\text { chuyên đề về IFRS }\end{array}$ & 7 & 21,88 \\
\hline Rất cao & 7 & 21,88 & $\begin{array}{l}\text { Tuyển giảng viên mới am } \\
\text { hiểu về IFRS, đồng thời } \\
\text { đào tạo giảng viên hiện tại }\end{array}$ & 8 & 25,00 \\
\hline Tổng cộng & 32 & 100 & Chưa có kế hoạch & 8 & 25,00 \\
\hline Mean $=3.59$, Std.Deviation $=1.012$ & & & & & \\
\hline
\end{tabular}

Kết quả khảo sát giảng viên cho thấy nhận định của họ về mức độ hiểu biết của lãnh đạo đơn vị đối với nhu cầu cần phải thay đổi CTĐT theo hướng tích hợp IFRS là tương đối cao $($ mean $=3.59)$. Trên $80 \%$ giảng viên cho rằng khoa đã có những bước chuẩn bị như phân công giảng viên giảng dạy IFRS, lập kế hoạch hỗ trợ kinh phí đào tạo cho giảng viên... Trên thực tế, các môn học có tích hợp một phần nội dung
Nguồn: Kết quả khảo sát của nhóm tác giả IFRS hiện phần lớn được quản lý bởi bộ môn Lý thuyết kế toán, tuy nhiên, Khoa Kế toán Kiểm toán đã chủ động phân công giảng viên ở cả ba bộ môn tham gia các khóa học đào tạo giảng dạy IFRS do ACCA Việt Nam tổ chức, đề nghị Trường hỗ trợ kinh phí cho giảng viên khi tham gia bồi dưỡng, học tập các chứng chỉ, lớp chuyên đề nâng cao liên quan tới môn học đang phụ trách giảng dạy.

\section{Bảng 13. Nhận thức của giảng viên, sinh viên về thách thức/rào cản của việc giảng dạy IFRS}

\begin{tabular}{|c|c|c|c|c|c|c|c|}
\hline \multicolumn{4}{|c|}{ Nhận thức về rào cản của giảng viên } & \multicolumn{4}{|c|}{ Nhận thức về rào cản của sinh viên } \\
\hline & $\mathrm{N}$ & Mean & \begin{tabular}{|c|} 
Std. \\
Deviation
\end{tabular} & & $\mathrm{N}$ & Mean & $\begin{array}{c}\text { Std. } \\
\text { Deviation }\end{array}$ \\
\hline $\begin{array}{l}\text { Thiết kế một phần riêng cho } \\
\text { IFRS trong CTĐT. }\end{array}$ & 32 & 3.44 & .982 & $\begin{array}{l}\text { Tài liệu học tập, } \\
\text { nghiên cứu IFRS }\end{array}$ & 142 & 3.28 & 1.041 \\
\hline $\begin{array}{l}\text { Phát triển tài liệu phục vụ } \\
\text { giảng dạy IFRS }\end{array}$ & 32 & 3.53 & 1.164 & $\begin{array}{l}\text { Trình độ giảng viên } \\
\text { giảng dạy IFRS }\end{array}$ & 142 & 3.44 & .993 \\
\hline Nguồn lực tài chính hỗ trợ & 32 & 4,00 & ,842 & Năng lực tiếng anh & 142 & 3,73 & ,974 \\
\hline $\begin{array}{l}\text { Đào tạo giảng viên giảng } \\
\text { dạy IFRS }\end{array}$ & 32 & 3,88 & 1,157 & \begin{tabular}{|l|l|}
$\begin{array}{l}\text { Quy mô lớp học } \\
\text { đông }\end{array}$ & \\
\end{tabular} & 142 & 3,67 & ,980 \\
\hline $\begin{array}{l}\text { Xác định thời điểm để bắt } \\
\text { đầu giảng dạy IFRS }\end{array}$ & 32 & 3,53 & 1,135 & & & & \\
\hline Chuyên gia về IFRS & 32 & 3,50 & ,803 & & & & \\
\hline Năng lực tiếng anh & 32 & 3,72 & 1,085 & & & & \\
\hline Quy mô lớp học đông & 32 & 3,91 & ,893 & & & & \\
\hline
\end{tabular}

Nguồn: Kết quả khảo sát của nhóm tác giả 
Bảng 13 cho thấy, giảng viên cho rằng thách thức, rào cản lớn nhất khi giảng dạy IFRS bao gồm nguồn lực tài chính hỗ trợ cho quá trình giảng dạy (mean $=4,00)$, quy mô lớp học đông (mean $=3,91)$, đào tạo giảng viên giảng dạy IFRS (mean $=3,88)$, và năng lực tiếng anh (mean $=3,72)$. Đối với sinh viên chưa nhận thức rõ được những thách thức sẽ gặp phải khi quá trình tích hợp IFRS trong giảng dạy diễn ra (mean < 4,0). Thách thức lớn nhất được sinh viên đánh giá là năng lực tiếng anh (mean = 3,73 ), kế tiếp là quy mô lớp học đông (mean $=3,67$ ). Quy mô lớp học đông là trở ngại lớn được nhận định bởi cả hai đối tượng giảng viên và sinh viên. Lớp học đông có thể gây ra những áp lực về mặt thời gian cho giảng viên để có thể bao quát đầy đủ các chủ đề, mục tiêu bài học đồng thời có thể tương tác, thảo luận với sinh viên. Đây cũng được xem là một trong những trở ngại lớn đối với người dạy và người học khi áp dụng các phương pháp dạy học hiện đại như phương pháp tình huống, học qua vấn đề và thực hành mô phỏng. Thực tế tại trường Đại học Tài chính - Marketing, ngoài các lớp hệ chất lượng cao, phần lớn các lớp hệ đại trà đang được giảng dạy với mô hình lớp học đông, trung bình 70 - 80 sinh viên/lớp. Điều này khiến giảng viên khó có thể tương tác với toàn bộ sinh viên cũng như theo dõi, đánh giá mức độ tiếp thu, khả năng thực hành của từng người học.
Nguồn lực tài chính hỗ trợ cùng với việc đào tạo giảng viên là hai trong những yếu tố mà đơn vị đào tạo cần xem xét khi tiến hành thay đổi CTĐT theo hướng tích hợp IFRS. Đặc điểm của IFRS là bộ chuẩn mực được thiết kế dựa trên các nguyên tắc. Do đó, thách thức cơ bản trong giảng dạy và đào tạo IFRS là việc người dạy ngoài trang bị tốt kiến thức để giảng dạy IFRS bằng phương pháp nguyên tắc (nhấn mạnh vào các khái niệm cơ bản thay vì các quy tắc hạch toán, giúp cho sinh viên có khả năng phát hiện và tự tìm ra các giải pháp), thì còn cần có thêm kinh nghiệm thực tế. Tuy nhiên, hầu hết các giảng viên kế toán hiện nay chưa đầu tư để có thêm các chứng chỉ nghề nghiệp quốc tế như ACCA, CPA, CIMA,... Trong đó, lý do về kinh phí đào tạo là một trong những nguyên nhân dẫn tới thực trạng này.

Rào cản về ngôn ngữ cũng là một trở ngại không nhỏ. IFRS được biên soạn bằng tiếng Anh; trong khi đó khả năng đọc, hiểu ngôn ngữ này của sinh viên và giảng viên còn nhiều hạn chế. Thêm vào đó, các giáo trình và tài liệu về IFRS bằng Tiếng Việt hiện nay hầu như không có và cũng không kịp cập nhật với những thay đổi của IASB. Điều này chính là trở ngại không nhỏ cho các giảng viên và sinh viên khi muốn đi sâu tìm hiểu về IFRS cũng như các văn bản hướng dẫn liên quan của bộ chuẩn mực này.

Bảng 14. Đánh giá mức độ hữu ích của tài liệu giảng dạy IFRS

\begin{tabular}{|c|r|r|r|r|r|r|}
\hline \multirow{2}{*}{ Nội dung } & \multicolumn{2}{|c|}{ Giảng viên } & \multicolumn{2}{c|}{ Sinh viên } & \multicolumn{2}{c|}{ Tổng cộng } \\
\cline { 2 - 7 } & \multicolumn{1}{c|}{ SL } & Tỷ lệ & SL & Tỷ lệ & SL & Tỷ lệ \\
\hline Câu hỏi và bài tập thực hành & \multicolumn{1}{c|}{2} & 6,25 & 22 & 15,49 & 24 & 13,79 \\
\hline Giáo trình & 17 & 53,13 & 41 & 28,87 & 58 & 33,33 \\
\hline Slides trình chiếu & 0 & 0,00 & 6 & 4,23 & 6 & 3,45 \\
\hline Tình huống thực tế & 13 & 40,63 & 72 & 50,70 & 85 & 48,85 \\
\hline Videos & 0 & 0,00 & 1 & 0,70 & 1 & 0,57 \\
\hline Tổng cộng & 32 & 100 & 142 & 100 & 174 & 100 \\
\hline
\end{tabular}

Nguồn: Kết quả khảo sát của nhóm tác giả 


\subsection{Hỗ trọ từ các tổ chức bên ngoài}

\section{Hoạt động CPD liên quan đến giảng dạy IFRS}

Kết quả nghiên cứu ở bảng 16 cho thấy chỉ $15,63 \%$ giảng viên chưa tham gia bất kỳ hoạt động CPD nào liên quan đến IFRS, 78,13\% giảng viên đã tham gia ít nhất một hội thảo về
IFRS, trong khi đó 36,5\% giảng viên đã từng tham gia các khóa học về IFRS. Tỷ lệ này còn khá thấp, do đó, giảng viên cần tham gia vào các chương trình CPD về IFRS nhiều hơn. Điều này đồng nghĩa với việc cần nhiều nỗ lực và sự tham gia từ các tổ chức học thuật, hiệp hội nghề nghiệp để hỗ trợ các sáng kiến thay đổi CTĐT tại các trường đại học.

Bảng 15. Nỗ lực cá nhân được thực hiện nhằm cập nhật và nâng cao kiến thức về IFRS

\begin{tabular}{|l|c|c|}
\hline \multicolumn{1}{|c|}{ Nội dung (Được lựa chọn nhiều phương án) } & \multicolumn{2}{|c|}{ Giảng viên } \\
\cline { 2 - 3 } & SL & Tỷ lệ \\
\hline Tham gia hội thảo về IFRS & 25 & $78,13 \%$ \\
\hline Tham gia học các khóa học về IFRS & 12 & $37,50 \%$ \\
\hline Tôi chưa tham gia bất kỳ hoạt động CPD nào liên quan đến giảng dạy IFRS & 5 & $15,63 \%$ \\
\hline
\end{tabular}

Nguồn: Kết quả phân tích dĩ liệu của nhóm tác giả

\section{Hoạt động hỗ trọ̣ khác}

\section{Bảng 16. Hoạt động hỗ trọ̣ khác của các tổ chức bên ngoài}

\begin{tabular}{|l|c|c|c|}
\hline & $\mathrm{N}$ & Mean & $\begin{array}{c}\text { Std. } \\
\text { Deviation }\end{array}$ \\
\hline $\begin{array}{l}\text { Cung cấp các tình huống nhằm so sánh, đối chiếu giữa IFRS và VAS } \\
\text { Việt Nam }\end{array}$ & 174 & 4.09 & .848 \\
\hline Cung cấp ngân hàng câu hỏi cùng đáp án & 174 & 4.06 & .806 \\
\hline Cung cấp các hội thảo về IFRS và giảng dạy IFRS & 174 & 4.09 & .848 \\
\hline Cung cấp chương trình đạo tạo giảng viên giảng dạy IFRS & 174 & 4.25 & .784 \\
\hline Cung cấp tài liệu giảng dạy IFRS phiên bản tiếng Việt & 174 & 4.22 & .782 \\
\hline Cung cấp chứng nhận về IFRS cho các CTĐT IFRS tại các trường đại học & 174 & 4.10 & .783 \\
\hline Cung cấp bản dịch chuẩn mực sang tiếng Việt & 174 & 4.20 & .898 \\
\hline
\end{tabular}

Nguồn: Kết quả phân tích dũ liệu của nhóm tác giả

Kết quả khảo sát cho thấy các giảng viên mong muốn các tổ chức bên ngoài sẽ đóng góp và cung cấp các dịch vụ hỗ trợ đào tạo IFRS (mean $>4.0$ ở tất cả các nội dung). Trong đó, nhu cầu về CTĐT giảng viên được đánh giá cao nhất (mean $=4 \cdot 25)$. Bên cạnh đó, các giảng viên hi vọng các tổ chức học thuật, hiệp hội nghề nghiệp có thể hỗ trợ cung cấp nguồn tài liệu giảng dạy IFRS phiên bản tiếng Việt (mean $=4.22$ ) và cung cấp bản dịch chuẩn mực (mean $=4.20)$.

\section{Kết luận và hàm ý chính sách}

\subsection{Kết luận}

Từ kết quả nghiên cứu trên cho thấy, giảng viên và sinh viên khoa Kế toán - Kiểm toán trường Đại học Tài chính - Marketing chuẩn bị sẵn sàng cho quá trình tích hợp IFRS vào CTĐT; đánh giá cao đối với các phương pháp sư phạm lấy người học làm trung tâm, phương pháp tình huống và phương pháp khuôn mẫu lý thuyết, các khái niệm của IFRS trước khi vào 
các nguyên tắc; các cấp lãnh đạo bộ môn và khoa có sự chuẩn bị tốt cho việc đào tạo giảng viên giảng dạy IFRS; và sự hỗ trợ của các tổ chức nghề nghiệp như ACCA, CPA, CIMA... trong việc đào tạo giảng viên giảng dạy IFRS, cung cấp tài liệu IFRS.

\subsection{Hàm ý chính sách}

Với thực trạng công tác đào tạo kế toán, kiểm toán của khoa Kế toán - Kiểm toán trường Đại học Tài chính - Marketing hiện nay, nhóm tác giả đề xuất một số giải pháp dựa trên cấu trúc mô hình CIF để có thể thúc đẩy quá trình tích hợp IFRS vào CTĐT chuyên ngành kế toán, kiểm toán như sau:

Hoàn thiện hồ sơ thự hiện thay đổi: Khoa KTKT-UFM cần xây dựng kế hoạch chuẩn bị, tăng cường tuyên truyền cho sinh viên về tính cấp thiết và lợi ích mà IFRS mang lại cho cơ hội nghề nghiệp trong tương lai thông qua các hội thảo, báo cáo chuyên đề, sinh hoạt nhóm... xây dựng CTĐT cần có sự tham chiếu, so sánh với các trường đại học trong nước và trên thế giới, và các tổ chức nghề nghiệp để tích hợp cao nhất các nội dung của IFRS.
Tăng cường nhận thức về phuoong pháp giảng dạy hiện đại: khoa Kế toán - Kiểm toán trường Đại học Tài chính - Marketing có thể tổ chức các lớp tập huấn để hướng dẫn giảng viên nhanh chóng chuyển đổi từ phương pháp giảng dạy kế toán dựa trên quy tắc như hiện nay sang phương pháp giảng dạy kế toán dựa trên nguyên tắc. Mời các chuyên gia đào tạo IFRS tập huấn cho giảng viên về đào tạo IFRS, hướng đến mô hình đào tạo kế toán gắn liền với IFRS trong thời gian sắp tới.

Tăng cường nguồn lục hỗ trợ đổi mới: Nâng cao năng lực tiếng Anh của giảng viên và sinh viên, có thể xem xét phương án sử dụng tiếng Anh làm ngôn ngữ giảng dạy chính bởi phần lớn tài liệu IFRS sẵn có được biên soạn bằng tiếng Anh, do đó có thể hiểu rõ được bản chất dựa trên nguyên tắc của IFRS mà không bị phụ thuộc vào chất lượng bản dịch.

Kết hợp hỗ trọ tù các tổ chức bên ngoài: khoa Kế toán - Kiểm toán trường Đại học Tài chính - Marketing cần có sự hợp tác chặt chẽ với các tổ chức nghề nghiệp như ACCA, CPA, CIMA... và được sự tài trợ của các tổ chức nghề nghiệp trong việc đào tạo giảng viên giảng dạy IFRS và nguồn tài liệu IFRS.

\section{TÀI LIÊU THAM KHẢO}

Albader, M. 2015. Transition to IFRS and its Implications for Accounting Education in Saudi Arabia, (May).

Alqahtani S. 2010.The Relevance of IFRS for Saudi Arabia: Stakeholders Perspective. PhD Thesis, Stirling University, UK.

Ball, DL \& Cohen, DK. 2000. 'Challenges of improving instruction: a view from the classroom', in T Duggan and M Holmes (eds), Closing the gap. Council for Basic Education, Washington, DC, pp. 28-38

Barnett, R \& Coate, K. 2005. Engaging the curriculum in higher education, The Society for Research Into Higher Education, McGraw-Hill Education, Maidenhead, Berkshire, England

Biggs, J. B. 2003. Teaching for Quality Learning at University. Society for Research into Higher Education \& Open University Press.

Biggs, J. B., \& Biggs, J. B. 1989. Higher Education Research \& Development Approaches to the Enhancement of Tertiary Teaching Approaches to the Enhancement of Tertiary Teaching, (March 2015), 37-41.

Bui, B., \& Porter, B. 2010. Accounting Education: An International The Expectation-Performance Gap in Accounting Education: An Exploratory Study The Expectation-Performance Gap in Accounting Education: An Exploratory Study, (March 2013), 37-41. https://doi.org/10.1080/09639280902875556

Carr, S., \& Mathews, M. R. 2004. Accounting curriculum change and iterative programme development : a case study Accounting curriculum change and iterative programme development: a case study, 9284 (December). https://doi.org/10.1080/0963928042000310814 
Coetzee, S. A., \& Schmulian, A. 2012. A Critical Analysis of the Pedagogical Approach Employed in an Introductory Course to IFRS, 27(1), 83-100. https://doi.org/10.2308/iace-10220

Çürük.T, Doğan.Z. 2002. Muhasebe ders ve içeriklerinin verilme düzeyleri konusunda işletmelerin üniversitelerden taleplerinin tespitine ilişkin bir araştırma Dokuz Eylül Üniversitesi IIBFF Dergisi, 17 (1) (2002), pp. 107-126

Feiter, L.D., Vonk, H., \& Akker, J.V. 1995. Towards more effective science teacher development in Southern Africa.

Fuller, A. 2007. Learning in Communities of Practice, Routledge, London.

Hargreaves, D.H., \&Hopkins, D. 1991.TheEmpowered School:The Management and Practice ofDevelopment Planning. London: Cassell Educational Limited. https://doi.org/10.1080/0924345930040305

Hilton, S. R., \& Johnstone, N. 2013. The IFRS transition and accounting education: A Canadian perspective post-transition. Issues in Accounting Education, 28(2), 253-261. https://doi.org/10.2308/iace-50366

Hodgdon, C., Hughes, S. B., \& Street, D. L. 2011. Framework-based Teaching of IFRS Judgements, (December 2014), 37-41. https://doi.org/10.1080/09639284.2011.589587

Howieson, B. 2003. Accounting practice in the new millennium : is accounting education ready to meet the challenge? (Vol. 35). https://doi.org/10.1016/S0890-8389(03)00004-0

IFRS. 2019. IFRS in your pocket 2019. Deloitte.

Jackling, B., Lange, P. A. De, \& Natoli, R. 2013. Transitioning to IFRS in Australian Classrooms: Impact on Teaching Approaches, 28(2), 263-275. https://doi.org/10.2308/iace-50358

KPMG.2011.KPMG-AAA2011 Survey, viewed12March2013,<http://www.kpmguniversityconnection.com/ IFRS/IFRS Faculty/ aaa-kpmg-survey2011. aspx?ReturnUrl= http:// www.kpmguniversityconnection. com/IFRS/IFRSFaculty/aaa-kpmg-survey-2011.aspx>.

Kutluk, F. A., Donmez, A., Utku, B. D., \& Erdogan, M. 2012. Expectation of Accounting Professionals From Accounting Education: An Antalya Research. Procedia - Social and Behavioral Sciences, 62, 418-423. https://doi.org/10.1016/j.sbspro.2012.09.068

Penuel, W. R., Fishman, B. J., Yamaguchi, R., \& Gallagher, L. P. 2007. What Makes Professional Development Effective? Strategies That Foster Curriculum Implementation. American Educational Research Journal, 44. https://doi.org/10.3102/0002831207308221

Pintó, R., Couso, D., \& Gutierrez, R. 2005. Using Research on Teachers 'Transformations of Innovations to Inform Teacher Education. The Case of Energy Degradation. https://doi.org/10.1002/sce.20042

Porter, AC, Garet, MS, Desimone, LM \& Birman, BF. 2003. 'Providing effective professional development: lessons from the Eisenhower Program', Science Educator, vol. 12, no. 1, pp. 23-40

Rebele, J. E. 2002. Accounting education's uncertain environments : descriptions and implications for accounting programmes and accounting education research. Accounting Education, 11(October 2014), 37-41. https://doi.org/10.1080/09639280210153245

Rogan, J. M., \& Grayson, D. J. 2003. Towards a theory of curriculum implementation with particular reference to science education in developing countries. International Journal of Science Education, 25(10), 1171-1204.

Singer, R. A. 2012. How to Integrate International. American Journal of Business Education, 5(3), 287-292.

Smylie,M.A.1997.FromBureaucratic ControltoBuildingHumanCapital :TheImportanceofTeacherLearning in Education Reform. Arts Education Policy Review, 99. https://doi.org/10.1080/10632919709600769

Wolf, P \& Hughes, JC. 2007. Curriculum development in higher education: faculty-driven processes and practices. Jossey-Bass Inc Pub, Wiley, United States

Yildiz, F., Durak, G. 2011. In the area of accounting education in universities BSNL Activity Expectations of Small and Medium-sized Enterprises. 49: 37-47 